\title{
Health Literacy and Its Determinants on Health Behavior among Mother with Overweight Children in Jakarta, Indonesia
}

\author{
Siti Syamsiah ${ }^{1}$, Nur Indrawati Lipoetoe ${ }^{2}$, Adnil Edwin Nurdin ${ }^{3}$, Adang Bachtiar ${ }^{4}$ \\ \{sitisyamsiah09@gmail.com ${ }^{1}$ \} \\ Department of Public Health, Universitas Andalas, Padang, Indonesia ${ }^{1,2,3}$ \\ Department of Public Health, Universitas Indonesia, Jakarta, Indonesia ${ }^{4}$ \\ Department of Public Health, Universitas MH Thamrin, Jakarta, Indonesia ${ }^{1}$
}

\begin{abstract}
Overweight children still serious problem in the world, which its prevalence among developing countries increases over the years. One of its risk factors is low health behavior (HB). Recently, one of important HB determinant is health literacy (HL), where low HL cause low HB. This study aims to analyze the influence of HL's determinant on HL and HB simultaneously among urban mother with overweight children. In This study, four HL's determinant, i.e., health status (HS), health education (HE), income status (IS) and social support (SS), will analyze its influence on HL and HB by using Structural Equation Model (SEM) approach with SmartPLS version 2. The design of the study is cross-sectional with 302 respondents of mothers in Jakarta that has children with $7-12$ years old. HL and its determinants have a significant effect on $\mathrm{HB}$ with confidence interval $(\mathrm{CI})=95 \%$. HS and $\mathrm{HE}$ has a significant effect with $\mathrm{CI}=95 \%$ both on $\mathrm{HL}$ and $\mathrm{HB}$. Meanwhile IS has a significant effect on $\mathrm{HL}$ and $\mathrm{HB}$ but with $\mathrm{CI}=80 \%$. SS has no significant effect on HL but has a significant effect on $\mathrm{HB}$ with $\mathrm{CI}=95 \%$. The effect of health literacy and its role if very important on health behavior. Although health literacy alone has a small effect in a matter of its magnitude to health behavior, however health literacy become moderating variable, which increases health literacy's determinant effect on health behavior.
\end{abstract}

Keywords: Health Behavior, Health Literacy, Health Literacy's Determinant, Overweight Children.

\section{Introduction}

Children who are overweight are considered to be one of the most serious public health problems in the world [1], where the number of overweight or obese infants and children (0 to 5 years) increased from 32 million globally in 1990 to 42 million in 2013 [2]. The prevalence of overweight among developing countries has increased over the years. The rate of obesity in Indonesia for toddlers is $11.9 \%$, while for adolescents aged 5-12 years $8.15 \%$ [3]. In Indonesia, the prevalence of obesity and obesity in toddlers is in the provinces of Lampung and South Sumatra, while for ages 5-12 years the highest prevalence is in DKI Jakarta Province with 30.1\% followed by Lampung by $29 \%$ [3].

Childhood obesity is a serious health problem that has adverse and long-term consequences for individuals, families, and communities [4]. Childhood obesity is related not only to the high 
risk of obesity brought to adulthood but also the increased risk of serious health conditions during childhood, including asthma, sleep apnea, bone, and joint problems, hypertension, high cholesterol, type 2 diabetes and psychological problems [5]. The high level and disparity is a concern, given that overweight children at 5 years of age are more likely to be obese later on (Ward et al., 2017). To reduce the risk of obesity for life, it is important that obesity prevention interventions begin before the age of 5 years [4].

One risk factor is low health behavior (HB), which is caused by low HB from their parents, especially their mothers, therefore obesity in children is strongly related to the role or function of health literacy in the elderly [6]. The increasing prevalence of obesity in children occurs one of them is because of the low ability of parents to obtain, manage, and understand basic health information and the services needed for proper health for their children to avoid obesity [7]. Children living in urban areas are more at risk than children who live in villages, mainly because children who live in urban areas are exposed to more types of ready-to-eat foods than children who live in rural areas [3].

Recently, one of the important determinants of HB is health literacy (HL), where low HL causes low HB, many causes of obesity, ultimately making a lot of research on the prevention of obesity, one of them with the Health Literacy model [8], explained that the concept of health literacy in health education and developing minimum health standards that are in school. However, a comprehensive study related to HL and the determining effect on HB among urban mothers with overweight children is still limited. This study aims to analyze the effect of HL determinants on HL and HB simultaneously among urban mothers with overweight children.

\section{Materials and Methods}

The study design was cross-sectional with 302 maternal respondents in Jakarta who had children aged 7 - 12 years. In this study, the inclusion and exclusion criteria were determined in such a way that the selected respondent was truly representing the population and its characteristics in accordance with the research objectives. The inclusion criteria are 1) mothers with complete families and 2) mothers who have smartphones and access to social media through smartphones. The exclusion criteria were: 1) mothers who had children who were not sick in the last 1 year and 2) mothers who had children who were not on a diet for the past 1 year.

In this study, four determinants of HL, namely: health status (HS), health education (HE), income status (IS) and social support (SS), will analyze the effect on HL and HB by using the Structural Equation Model (SEM Structural Equation Model). The SEM that was conducted in this study used a variance-based SEM approach using SmartPLS version 2 software. This approach was used because of the consideration of flexibility and predictive ability of variancebased SEM.

In developing the model, each indicator developed indicators that represent the variable. Later in the SEM process, each of these indicators will be evaluated to what extent they represent the variables and then simultaneously these variables are evaluated as well as the extent to which they relate to other variables. Fig 1 is a model of the relationship between variables and variables with indicators that will be used in this study. 


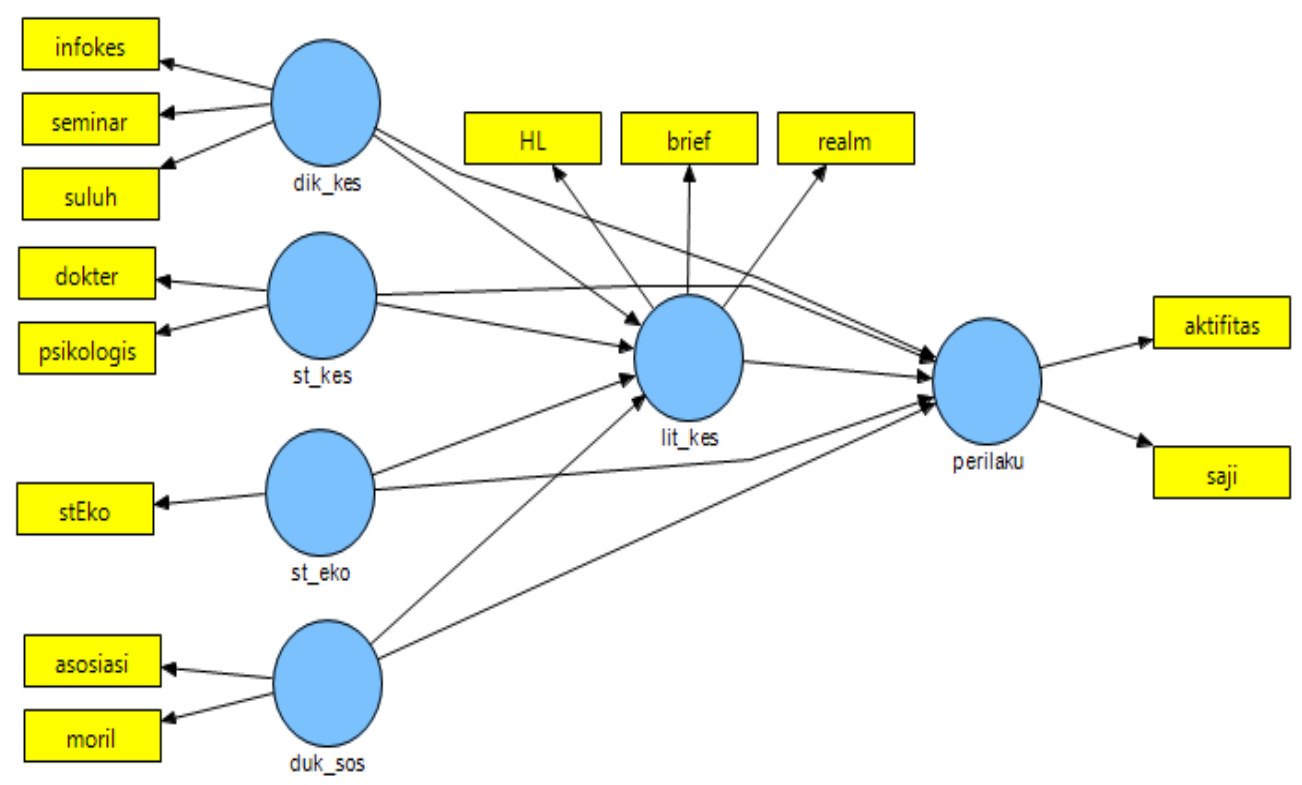

Fig. 1. Model SEMAwal

Where, dik_kes (HE), st_kes (HS), st_eko (IS), duk_sos (SS), lit_kes (HL) and behavior (HB). 3 indicators, namely represent him: 1) invokes (health information), 2) seminars (number of health seminars followed) and 3) torches (the number of health counseling followed. While 2 indicators, namely: 1 represent HS) doctor ( ease of visit to the doctor) and 2) psychological (psychological health). For IS, it is only represented by one indicator which is the questions about the ability and economic conditions of the respondents. While for HL is represented by: 1) HL (collection of questions about health litersion), 2) brief (containing a list of health terms) and 3) realm (condition of respondents' health literacy knowledge). The last for variable HB is represented by 2 indicators, they are: 1) activity (containing physical activity) and 2) serving (containing questions about serving daily food).

\section{Results}

From the results of SEM analysis, several indicators are discarded and the relationship lines between variables are discarded because the relationship is not significant. Fig 2 displays the final model after several indicators and relationships between variables are discarded. 


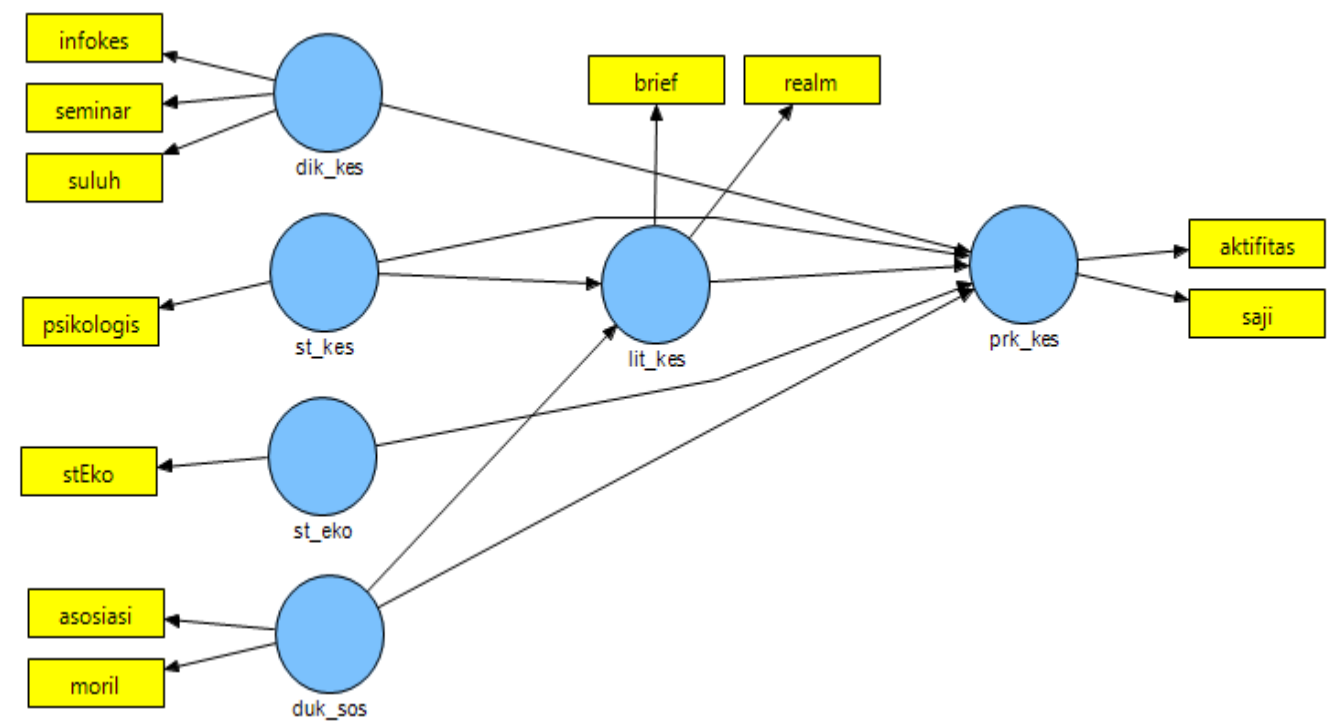

Fig. 2. Model SEM Akhir

From Fig. 2, it is known that the physician and HL indicators are removed and the relationship between dik_kes (HE) and lit_kes (HL) is discarded, and the relationship between st_eko (IS) and lit_kes (HL) is also discarded. It shows that the relationship between HE and HL is not significant in the context of this research and IS also has no significant relationship to HL.

$\mathrm{HL}$ and its determinants have a significant effect on $\mathrm{HB}$ with a confidence interval $(\mathrm{CI})=$ 95\%. HS and HE have a significant effect with $\mathrm{CI}=95 \%$ on both HL and HB. Meanwhile, IS has a significant influence on $\mathrm{HL}$ and $\mathrm{HB}$ but with $\mathrm{CI}=80 \%$. SS did not have a significant effect on HL but had a significant effect on $\mathrm{HB}$ with $\mathrm{CI}=95 \%$. In the case of effect size or effect size; HL, HS and IS have a small effect on HB (f2 $\leq 0.02$ ). While HE has a moderate effect size on $\mathrm{HB}$ (f $2 \geq 0.15$ ). Even though HL has a small effect on HB, the determinant of HL has increased the total effect on HB by $5.38 \%, 14 \%$ and $15 \%$ for HE, HS and IS respectively.

\section{Discussion}

$\mathrm{HL}$ and its determinants have a significant effect on $\mathrm{HB}$ with a confidence interval $(\mathrm{CI})=$ $95 \%$. This is consistent with research that explains that there is significant influence between health literacy (health literacy) and health behavior [9]. Health literacy in this context is the extent to which individuals achieve, manage, and understand health information and apply that information in health decision making [10]. Regarding this context, there are three types of health literacy: functional, communicative/interactive, and critical health literacy [10]. Functional health literacy is the application of literacy and numeracy skills for health-related material such as food and beverage labels consumed. The communicative/interactive health literacy is the application of cognitive functions and social skills to actively participate in understanding various forms of communication and applying information new to developing situations related to health information, Meanwhile, the critical health literacy empowers 
individuals to act on social, economic and environmental determinants of health through individual and collective efforts of the most developed and understood aspects of health literacy related to the prevention of obesity in children [11]. HS and HE have a significant effect with $\mathrm{CI}=95 \%$ on both HL and HB.

\section{Conclusions}

The influence of health literacy and its role is very important in health behavior. Although health literacy alone has little effect on the magnitude of health behavior, health literacy is a moderating variable, which increases the determinants of health literacy in health behavior.

\section{References}

[1] Black, N., Hughes, R. and Jones, A. M. (2018) 'The health care costs of childhood obesity in Australia: An instrumental variables approach', Economics and Human Biology. Elsevier B.V., 31, pp. 1-13. doi: 10.1016/j.ehb.2018.07.003.

[2] Ward, D. S. et al. (2017) 'Strength of obesity prevention interventions in early care and education settings: A systematic review', Preventive Medicine. Elsevier B.V., 95, pp. S37-S52. doi: 10.1016/j.ypmed.2016.09.033.

[3] Penelitian, B. and Pengembangan, D. A. N. (2010) 'RISET KESEHATAN DASAR'

[4] Leann, L., Parker, L. and Burns, A. (2011) Early Childhood Obesity Prevention Policies. doi: $10.17226 / 13124$.

[5] Reilly, J. J. et al. (2003) 'Health consequences of obesity.', Arch Dis Child, 88(9), pp. 748-52. doi: 10.1136/ADC.88.9.748.

[6] Chari, R. et al. (2014) 'Association between health literacy and child and adolescent obesity', Patient Education and Counseling. Elsevier Ireland Ltd, 94(1), pp. 61-66. doi: 10.1016/j.pec.2013.09.006.

[7] Conner, M. and Sparks, P. (2005) 'Theory of planned behaviour and health behaviour', Predicting health behaviour: Research and practice with social cognition models, pp. 170-222.

[8] WHO (2008) 'Education material for teachers of midwifery', Midwifery education modules, 12, p. 75

[9] Fleary, S. A., Joseph, P. and Pappagianopoulos, J. E. (2018) 'Adolescent health literacy and health behaviors: A systematic review', Journal of Adolescence. Elsevier, 62(October 2017), pp. 116-127. doi: 10.1016/j.adolescence.2017.11.010.

[10] Parker, R. M., Ratzan, S. C. and Lurie, N. (2003) 'Health literacy: A policy challenge for advancing high-quality health care', Health Affairs, 22(4), pp. 147-153. doi: 10.1377/hlthaff.22.4.147.

[11] Sykes, S. et al. (2013) 'Understanding critical health literacy: A concept analysis', BMC Public Health, 13(1). doi: 10.1186/1471-2458-13-150. 